\title{
Application of Molecular Imprinting Technique in Organophosphorus Pesticides Detection
}

\author{
Liu Zhao ${ }^{1,2}$, Hua Ping ${ }^{1,2}$, Ling Xiang ${ }^{1,2}$, Ping Han $^{1,2}$, \\ Jihua Wang ${ }^{1,2}$, and Ligang Pan $^{1,2, *}$ \\ ${ }^{1}$ Beijing Research Center for Agrifood Testing and Farmland Monitoring, \\ 100097 Beijing, China \\ ${ }^{2}$ Beijing Research Center for Information Technology in Agriculture, 100097 Beijing, China \\ Tel.: +86 10 51503031; Fax: +86 1051503406 \\ \{Liu. Zhao, Hua. Ping, Ling.Xiang, Ping. Han, \\ Jihua.Wang, Ligang. Pan, zhaoliu\}@nercita.org.cn, \\ panlg@nercita.org.cn
}

\begin{abstract}
Molecular imprinting technique offers a means of producing practical materials that are able to recognize a certain molecule in terms of shape, size and chemical functionality. In order to obtain a highly selective recognition of organophosphorus pesticides (OPPs), we synthesized molecularly imprinted polymers (MIPs) using pirimiphos-methyl as the template, methacrylic acid as the monomer and ethylene glycol dimethacrylate as the crosslinker. After polymerization, molecularly imprinted solid-phase extraction (MISPE) was used for the selective preconcentration of OPPs. The preparation methods and synthesis conditions of MIPs were discussed, and the specificity of MIPs and nonimprinted polymers were investigated. The results showed that MIPs enable the selective extraction of pirimiphos-methyl successfully from water sample, and demonstrated the potential of MISPE for selective and cost-effective sample pretreatment.
\end{abstract}

Keywords: Molecularly imprinted polymers, Solid phase extraction, Organophosphorus pesticides.

\section{Introduction}

Organophosphorus pesticides (OPPs) have often been employed in farmland cultivation over the last several decades and are still continuously used in modern agricultural systems[1]. These OPPs exhibit acute or chronic toxicity to human, environment and the biota thus emphasizing the need for efficient analytical procedures to monitor potential risks. Most OPPs are easily analyzed by GC and HPLC. Generally, the trace analysis needs a pretreatment step in order to reduce the matrix interference and enrich the analyte. This is often performed by solid-phase extraction (SPE) [2-4].

Molecular imprinting is a versatile technique that creates molecular assemblies of desired chemical structures and properties[5]. During last decade, molecularly

\footnotetext{
* Corresponding author.
} 
imprinted polymers (MIPs) have demonstrated a great potential as selective sorbents and have been widely used for the clean-up of samples in SPE processes[6-8], namely molecularly imprinted solid-phase extraction (MISPE). The ongoing research has proven that MIPs can be efficiently used in this field[9-10].

In this article, we reported the synthesized method of acrylic-based MIPs following the non-covalent approach and the selective use of MISPE technique for the analysis of organophosphorus pesticides.

\section{Materials and Methods}

\subsection{Materials}

Pirimiphos-methyl (99.7\%), methylnitrophos (99.2\%) and malathion (97.2\%) were purchased from $\mathbf{J} \& \mathrm{~K}$ Scientific (Beijing, China). Methacrylic acid (MAA) and ethylene glycol dimethacrylate (EGDMA) were purchased from Sigma. The initiator 2,2'-azobisisobutyronitrile (AIBN) was purchased from Shanghai Chemical Plant (Shanghai, China). All solvents of analytical grade were purchased from Beijing Chemical Reagent (Beijing, China). All syntheses were carried out using distilleddeionized water (18.2 M $\Omega . \mathrm{cm}$, PALL system). All solutions were filtered through a $0.45 \mu \mathrm{m}$ membrane from Millipore before use.

\subsection{Polymer Preparation}

The template pirimiphos-methyl $(0.305 \mathrm{~g}, 1 \mathrm{mM})$ and functional monomer MAA $(0.344 \mathrm{~g}, 4 \mathrm{mM})$ were dissolved in $5.6 \mathrm{~mL}$ dichloromethane in a $40-\mathrm{mL}$ glass tube with slightly shaking for $6 \mathrm{~h}$. To this solution, cross-linker EGDMA (3.964g, 20mM) and AIBN (40mg, $0.24 \mathrm{mM}$ ) were added in steps. Then, the mixture was deoxygenated with nitrogen for $15 \mathrm{~min}$, followed by degasification under vacuum for $5 \mathrm{~min}$ and sealed. The polymerization was carried out by heating the mixture in a $60^{\circ} \mathrm{C}$ water bath for $36 \mathrm{~h}$. The obtained polymer was ground to fine powders and sieved to obtain $60-70 \mu \mathrm{m}$ particles. These particles were extracted in a Soxhlet for $24 \mathrm{~h}$ with methanolacetic acid (9:1, v/v) until no residue of template was found in the rinses.

The corresponding non-imprinted polymers (NIPs) for comparison experiments were prepared in the same manner but without addition of template.

\subsection{MISPE Protocol}

Two hundred milligrams of the cleaned-up MIPs (or NIPs) were put into a $10 \mathrm{~mL}$ vial and incubated with methanol, standing at ambient temperature with occasional shaking for $24 \mathrm{~h}$. Then the slurry was transferred into a $3 \mathrm{~mL}$ polypropylene SPE cartridge and stood for $30 \mathrm{~min}$. After that, polyethylene frit was carefully put onto the polymer to stabilize the sorbents. MIPs of the size $60-70 \mu \mathrm{m}$ proved to be an acceptable compromise between homogeneity and permeability of SPE cartridge. Prior to use, the MIPs (or NIPs) SPE-cartridges were conditioned by washing with $10 \mathrm{~mL}$ methanol-acetic acid (9:1, v/v) and $2 \mathrm{~mL}$ methanol, followed by $2 \mathrm{~mL}$ water. For the MISPE process, standard solution (a mixture of pirimiphos-methyl, methylnitrophos and 
malathion, $1 \mu \mathrm{g} / \mathrm{mL}$ ) and a spiked water sample were loaded onto the MISPE cartridges at a flow rate of $0.4 \mathrm{~mL} / \mathrm{min}$ respectively. And then the SPE cartridges were washed with $2 \mathrm{~mL}$ dichloromethane/acetonitrile $(95: 5, \mathrm{v} / \mathrm{v})$ and eluted with $2 \mathrm{~mL}$ dichloromethane/methanol $(90: 10, \mathrm{v} / \mathrm{v})$ by steps. The eluate was immediately dried under a stream of nitrogen, and the residue was dissolved in $1 \mathrm{~mL}$ dichloromethane for GC-MS analysis.

\subsection{GC-MS Assay}

The assay was conducted by Shimadzu GC-MS QP2010 Plus. The analyses were carried out on a gas chromatograph fitted with a HP-5 MS capillary column (30 $\mathrm{m} \times 0.25 \mathrm{~mm}$ id; $0.25 \mu \mathrm{m}$ film thickness). Analytical gas chromatography conditions were as follows: injector temperature $230^{\circ} \mathrm{C}$; oven temperature held at $120^{\circ} \mathrm{C}$ for 5 min, then programmed to increase from $120^{\circ} \mathrm{C}$ to $150^{\circ} \mathrm{C}$ at a rate of $5^{\circ} \mathrm{C} / \mathrm{min}$ and held for $7 \mathrm{~min}$; carrier gas, helium at a flow rate of $1 \mathrm{~mL} / \mathrm{min}$; Mass spectrometer conditions: ionization mode with $\mathrm{EI}$, electron energy $70 \mathrm{eV}$, ion source temperature $230^{\circ} \mathrm{C}$, interface temperature $220^{\circ} \mathrm{C}$. Instrument operation and data processing was done through the LabSolutions (version 2.50) software.

\section{Results and Discussion}

\subsection{Synthesis of Polymer}

The choice of polymerisation solvent is the key point of the adduct formation and the promotion of the imprinting efficiency[11]. Dichloromethane is one of the most widely used solvents, since it satisfactorily dissolves all the reaction components and does not suppresses hydrogen bonding. We speculate that the use of dichloromethane can enhance the unspecific binding of analyte to the crosslinker. In addition, the nature of the crosslinker is another key factor of the polymer specificity[12]. The reactivity of the crosslinker should be similar to that of the functional monomer. And the mole ratios of crosslinker to functional monomer are also important[13]. Generally, hydrogen bonding is dependent on both distance and direction between monomers and templates. EGDMA as the shortest crosslinker led to the highest selectivity in the polymer[14].

\subsection{Specificity of Polymer}

One merit of MISPE is that the polymer sorbents have good selectivity for the template molecule. To evaluate the specificity of this kind of SPE materials, the molecular recognition properties of three different OPPs (pirimiphos-methyl, methylnitrophos, malathion) was investigated. A total of $1.0 \mathrm{~mL}$ of a mixture of $1 \mu \mathrm{g} / \mathrm{mL}$ of each organophosphorus was applied to the MIP and blank polymer cartridges, and then the compounds in both the washing and elution fractions were analyzed by GC-MS. 


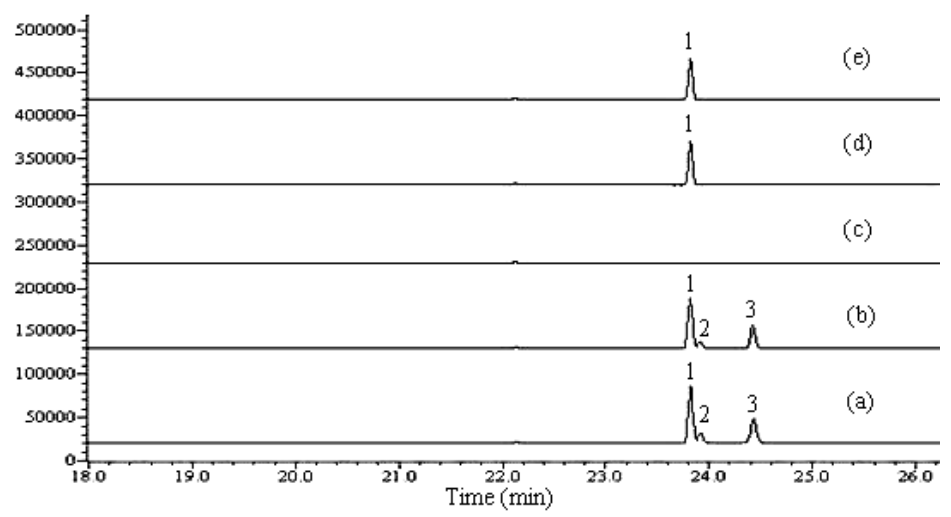

Fig. 1. Chromatograms obtained by off-line SPE of $1.0 \mathrm{~mL}$ of a mixture of $1 \mu \mathrm{g} / \mathrm{mL}$ of each OPP. (a) standard solution; (b) NIP, washing fraction; (c) NIP, elution fraction; (d) MIP, elution fraction; (e) water sample. (1) pirimiphos-methyl; (2) methylnitrophos; (3) malathion. Wash step: $2 \mathrm{~mL}$ dichloromethane/acetonitrile $(95: 5$, v/v). Elution step: $2 \mathrm{~mL}$ dichloromethane/methanol (90:10, v/v).

Fig.1 showed the chromatograms of OPPs in standard solution, washing solutions, and elution fractions. It can be seen that almost all of the OPPs were completely removed from the blank column after the washing step. However, a different result was observed for the MISPE cartridge. Pirimiphos-methyl, the template molecular, was still totally retained on the MISPE column after the washing step. The recovery of pirimiphos-methyl was higher than $80 \%$. In addition, the left OPPs were not retained on the MISPE column. In other words, they cannot be recognized by the MIPs and were completely separated from the target analyte. These results showed that the MIP exhibited highly selective binding affinity for pirimiphos-methyl and no binding for the left OPPs. Although there is only slight difference between the structures of those three OPPs, this further explains that the imprinting is not only based on the interaction of the functional groups of the analyte but also based on the combined effect of shape and size complementarily ${ }^{[15]}$. The recovery of three OPPs (Table 1) showed that the MIP cartridge could be proved to be a powerful tool for the enrichment of pirimiphos-methyl.

\subsection{Determination of Pirimiphos-Methyl in Spiked Water Sample}

To demonstrate the applicability of reliability of this method for environmental application, real environmental water sample was selected and analyzed. Tap water was spiked with pirimiphos-methyl at the $1 \mu \mathrm{g} / \mathrm{mL}$ concentration level and was preconcentrated by MISPE. The recovery and reproducibility of this method were calculated and summarized in Table 1. As expected, for analysis of pirimiphos-methyl in the water sample, the analyte recovery was higher than $80 \%$. 
Table 1. Recoveries of three OPPs after loading of $1.0 \mathrm{~mL}$ of $1 \mu \mathrm{g} / \mathrm{mL}$ of each OPP onto the SPE cartridges $(n=3)$

\begin{tabular}{ccccc}
\hline \multirow{2}{*}{ Analyte } & \multicolumn{2}{c}{ NIP $(\% \pm \mathrm{SD})$} & \multicolumn{2}{c}{$\mathrm{MIP}(\% \pm \mathrm{SD})$} \\
\cline { 2 - 5 } & Washing & Elution & Elution & Tap water \\
\hline Pirimiphos-methyl & $97.6 \pm 2.5$ & 0 & $83.2 \pm 3.1$ & $80.1 \pm 2.3$ \\
Methylnitrophos & $96.1 \pm 3.9$ & 0 & 0 & 0 \\
Malathion & $99.3 \pm 2.6$ & 0 & 0 & 0 \\
\hline
\end{tabular}

\section{Conclusion}

In this report, we discussed the utility of molecular imprinting technology for the organophosphorus pesticides detection. MIPs selective for pirimiphos-methyl was prepared and applied as the material for SPE in off-line separations. The polymer showed well affinity and selectivity to pirimiphos-methyl. And the MISPE proved to be an effective tool for the enrichment of pirimiphos-methyl from water sample. For organophosphorus pesticides, the MISPE approach provided simpler methodology and significant increases in selectivity relative to the conventional methods. And we feel that this approach will be a useful analytical tool for analyzing complex samples, especially in performing the initial screening of libraries against poorly characterized receptors.

Acknowledgments. This work was supported by the National High Technology Research and Development Program 863 (2010AA10Z403, 2007AA10Z202) and Beijing Municipal Science and Technology Commission Program (Z09090501040901).

\section{References}

1. Liu, J., Wang, L., Zheng, L., Wang, X., Lee, F.S.: Analysis of bacteria degradation products of methyl parathion by liquid chromatography/electrospray time-of-flight mass spectrometry and gas chromatography/mass spectrometry. J. Chromatogr. A. 1137(2), 180-187 (2006)

2. Brito, N.M., Navickiene, S., Polese, L., Jardim, E.F., Abakerli, R.B., Ribeiro, M.L.: Determination of pesticide residues in coconut water by liquid-liquid extraction and gas chromatography with electron-capture plus thermionic specific detection and solid-phase extraction and high-performance liquid chromatography with ultraviolet detection. J. Chromatogr. A. 957(2), 201-209 (2002)

3. Ismail, N., Vairamani, M., Rao, R.N.: Determination of cis and trans isomers of monocrotophos in technical products by reversed-phase column liquid chromatography. J. Chromatogr. A. 903(1-2), 255-260 (2000)

4. Mullett, W.M., Lai, E.P.C.: Determination of theophylline in serum by molecularly imprinted solid-phase extraction with pulsed elution. Anal. Chem. 70, 3636-3641 (1998)

5. Hong, C.C., Chang, P.H., Lin, C.C., Hong, C.L.: A disposable microfluidic biochip with on-chip molecularly imprinted biosensors for optical detection of anesthetic propofol. Biosens. Bioelectron. 25, 2058-2064 (2010) 
6. Pichon, V., Haupt, K.: Affinity separations on molecularly imprinted polymers with special emphasis on solid- phase extraction. J. Liq. Chromatogr. Rel. Technol. 29(7-8), 989-1023 (2006)

7. Caro, E., Marce, R., Borrull, F., Cormack, P.A.G., Sherrington, D.C.: Application of molecularly imprinted polymers to solid-phase extraction of compounds from environmental and biological samples. Trends. Anal. Chem. 25, 143-154 (2006)

8. Andersson, L.I.: Molecular imprinting for drug bioanalysis. J. Chromatogr. B. 739, 163-173 (2000)

9. Ramstrom, O., Skudar, K., Haines, J., Patel, P., Bruggemann, O.: Food analyses using molecularly imprinted polymers. J. Agric. Food. Chem. 49, 2016-2114 (2001)

10. Bereczki, A., Tolokan, A., Horvai, G., Horvath, V., Lanza, F., Hall, A.J., Sellergren, B.: Determination of phenytoin in plasma by molecularly imprinted solid-phase extraction. J. Chromatogr. A. 930(1-2), 31-38 (2001)

11. O'Shannessy, D.J., Ekberg, B., Mosbach, K.: Molecular imprinting of amino-acid derivatives at low-temperature (0-degrees-C) using photolytic homolysis of azobisnitriles. Anal. Biochem. 177(1), 144-149 (1989)

12. Wulff, G., Vietmeier, J., Poll, H.G.: Enzyme-analogue built polymers. 22. Influence of the nature of the crosslinking agent on the performance of imprinted polymers in racemic resolution. Makromol. Chem. 188, 731-740 (1987)

13. Ansell, R.J., Mosbach, K.: Molecularly imprinted polymers by suspension polymerization in perfluorocarbon liquids, with emphasis on the influence of the porogenic solvent. J. Chromatogr. A. 787, 55-66 (1997)

14. Wulff, G., Vesper, W.: Preparation of chromatographicsorbents with chiral cavities for racemic resolution. J. Chrom. 167, 171-186 (1978)

15. Lu, Y., Li, C., Liu, X., Huang, W.: Molecular recognition through the exact placement of functional groups on non-covalent molecularly imprinted polymers. J. Chromatogr. A. 950(1-2), 89-97 (2002) 